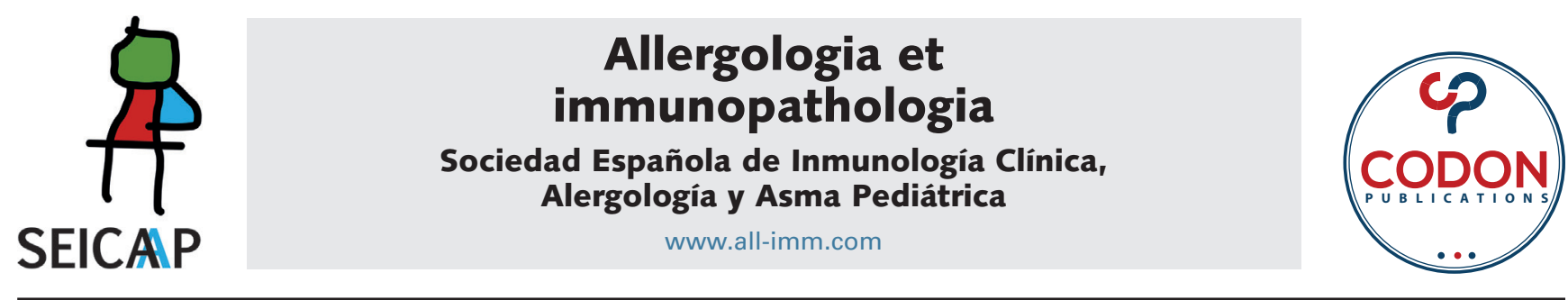

REVIEW ARTICLE

OPEN ACCESS (C) (1) (2)

\title{
Desensitization to drugs in children
}

\section{Luis Felipe Ensina ${ }^{a *}$, Mara Morelo Rocha Felix ${ }^{\mathrm{b}}$, Fernanda Sales da Cunha ${ }^{\mathrm{b}}$, Jean-Christoph Caubet ${ }^{c}$}

\author{
${ }^{a}$ Department of Pediatrics, Federal University of São Paulo (EPM-UNIFESP), São Paulo, Brazil \\ ${ }^{b}$ Department of General Medicine, Universidade Federal do Estado do Rio de Janeiro, Rio de Janeiro, Brazil \\ 'Department of Child and Adolescent, Geneva University Hospitals, Geneva, Switzerland
}

Received 9 November 2021; Accepted 5 January 2022

Available online 1 March 2022

\section{KEYWORDS}

adolescent;

children;

desensitization;

drug allergy;

drug hypersensitivity;

pediatric

\begin{abstract}
Better knowledge and understanding about drug desensitization is required in the pediatric population, since there is little literature available about it and the most pediatric desensitization protocols have been adapted from adult instructions.

Aiming to soften this issue and foster the future studies, this article presents a recent review about mechanisms of desensitization, diagnostic tools, and up to date management of drug hypersensitivity reactions in children. Bringing up an overview of pediatric hypersensitivity reactions to chemotherapy, biologic agents, antibiotics, nonsteroidal anti-inflammatory drugs, and vaccines.
\end{abstract}

(c) 2022 Codon Publications. Published by Codon Publications.

\section{Introduction}

Drug hypersensitivity reactions (DHR) are adverse drug reactions that resemble allergic manifestations. When immune mechanisms mediate these reactions, they are called drug allergies.,2 Drug allergies can be classified according to their mechanism in ${ }^{3}$ :

- Type I (Immunoglobulin E [IgE]-mediated reactions)

- Type II (antibody-mediated cytotoxicity reactions)

- Type III (immune complex-mediated reactions)

- Type IV for delayed-type hypersensitivity - Type IVa (Th1)
- Type IVb (Th2)

- Type IVc (Cytotoxic T cells)

- Type IVd (T cells)

The most frequent reactions caused by drugs are type I and IV reactions. ${ }^{2}$ Type I reactions are classically immediate reactions that appear within an hour and up to $6 \mathrm{~h}$ after administration of a drug and manifest as urticaria, angioedema, rhinitis, wheezing, gastrointestinal symptoms, or anaphylaxis. Type II reactions can occur 5-15 days after drug administration and include manifestations such as anemia or thrombocytopenia caused by drugs. Type III reactions initiate 7-21 days after drug administration, and

*Corresponding author: Luis Felipe Ensina, Department of Pediatrics, Federal University of São Paulo (EPM-UNIFESP), São Paulo, Brazil. Email address: 100alergia@gmail.com 
examples are serum sickness-like disease or vasculitis. Type IV reactions have a more heterogeneous presentation, and appear in days, weeks, or months after initiation of a drug. They are mediated by $T$ cells and may have a benign presentation such as a maculopapular exanthema or a poor prognosis as observed in patients of severe cutaneous adverse reactions (SCAR), which include Stevens-Johnson Syndrome (SJS) and toxic epidermal necrolysis (TEN), drug reaction with eosinophilia and systemic symptoms (DRESS), and acute generalized exanthematous pustulosis (AGEP). ${ }^{2,4}$

Clinically, DHR can be classified as immediate or non-immediate (mediated reactions). Immediate reactions may have an IgE or non-lgE-mediated mechanism and occur within 1-6h after the last drug administration. Nonimmediate reactions may occur any time as from $1 \mathrm{~h}$ after the initial drug administration. They commonly appear after many days of treatment and are often associated with a delayed $\mathrm{T}$ cell-dependent type of allergic mechanism. ${ }^{2}$ There is an overlap between immediate and nonimmediate reactions regarding the time interval between drug administration and the onset of signs and symptoms.

In drug allergy, phenotypes are based on the clinical characteristics of patients and symptoms, and the time of onset of manifestations after drug administration. ${ }^{4}$ Endotypes are related to the pathogenic mechanisms of reactions. In order to understand these mechanisms, in vivo and in vitro tests are required, such as skin test (ST), specific IgE, and basophil activation test (BAT), which helps to identify the involvement of mast cells and basophils in DHR. Skin test and specific IgE clarify the involvement of any lgE-dependent pathway. BAT is another interesting diagnostic tool and potential biomarker for basophil activation, evaluating the expression of $\mathrm{CD} 203 \mathrm{C}$ and CD63 on basophils. ${ }^{4-6}$

Children with severe diseases such as cancer, autoimmune diseases, or cystic fibrosis can become allergic to their first-line therapy after repeated exposures. ${ }^{4}$ Avoiding first-line treatment because of hypersensitivity can affect these patients' quality of life and survival. ${ }^{2}$ Drug desensitization (DS) is a procedure designed to safely reintroduce drugs into patients who have had IgE/non-IgE type I reactions. ${ }^{4,7}$

Drug desensitization is defined as the induction of a temporary state of tolerance of a drug for a hypersensitivity reaction. It is performed by administering increasing doses of medication over a short period (from several hours to a few days) until the total cumulative therapeutic dose is achieved and tolerated. ${ }^{4,7}$

Knowledge of the severity of reaction, the molecular mechanisms involved in DHR, and the patient's comorbidities could help in risk stratification during the procedure. Thus, it would be possible to determine whether drug desensitization is indicated and the potential risk of reaction during the procedure. ${ }^{4}$

\section{Mechanisms of desensitization}

Desensitization procedures primarily involve mast cells and basophils, as these are the cells that degranulate during DHR and drug desensitization. ${ }^{4}$ Studies have demonstrated that mast cells become unresponsive to the implicated drug after drug desensitization. ${ }^{8}$ Mast cells can be activated through IgE or non-IgE-dependent pathways. Small molecule drugs may induce systemic non-IgE-mediated reactions by activating human Mas-related guanine nucleotide-binding (G)-protein coupled receptor member X2 (MRGPRX2). ${ }^{9}$

Many studies have demonstrated the inhibition of activation hallmarks of mast cells during DS protocols. In vitro models demonstrate that compared to activated cells, desensitized cells had a diminished immediate release of $\beta$-hexosaminidase, early and late tumor necrosis factor- $\alpha$ (TNF- $\alpha$ ), Interleukin 6 (IL-6) production, de novo synthesis of lipid mediators, and calcium flux..$^{8,10,11}$ These studies have indicated that both time and dose are critical for degranulation of mast cells..$^{8,10}$ The interval at which the drug is administered is crucial. Another relevant aspect is the infusion rate. Similarly, the dose must be ideal to avoid breakthrough reactions.

One possible mechanism implies that increasing sub-therapeutic doses can supply a sufficient number of antigenic determinants to bind to IgE anchored to the surface Fc epsilon RI (Fc\&RI) receptors but not to cross-link such IgE. Alternatively, the antigen can induce rapid internalization of cross-linked antigen receptors, which depletes the cell surface of these receptors, preparing the cell nonreactive to the antigen. ${ }^{12}$

More recent studies have demonstrated that antigen/ IgE/Fc\&RI complexes' internalization is impaired. Compared to activated cells, where most are internalized, these complexes remain on the surface during drug desensitization. In IgE-mediated mast cell activation, the antigen valency and dose have been demonstrated to be critical factors directly affecting Fc\&RI behavior. ${ }^{8,10}$

Another possible explanation is that in the early stages of drug desensitization, the inhibitory pathway of immunoreceptor tyrosine-based inhibitory motif (ITIM) is dominant whereas in the later stages, it replaces activation signals. More studies are required to identify the ITIM and phosphatases that regulate immunoreceptor tyrosine-based activation motif (ITAM) during drug desensitization. ${ }^{4}$ It is also possible that degradation of signal molecules such as Syk and Lyn takes place. In vitro drug desensitization of human mast cells depicts the degradation of these molecules. ${ }^{13}$

A small amount of intracellular calcium mobilization is induced by sub-threshold antigen doses during drug desensitization. Low antigen doses may create a continuous low intracellular calcium level, which causes conformational changes in calcium-related channels. Further calcium entry and signal transduction would be blocked by these modifications in the receptors. $8,10,11$

Finally, recent studies have established that actin cytoskeleton participates in calcium mobilization in mast cells. Mast cells' lack of response could be mediated by the stable remodeled actin cytoskeleton required to compartmentalize desensitized receptors. , $^{8,10}$

\section{Indications and contraindications}

Drug desensitization is a therapeutic procedure indicated for patients with proven or highly suspected DHR. It must 
be recommended after an assessment of individual risk versus benefits, indicating that the benefits outweigh the risks. ${ }^{13}$

Following are the indications of drug desensitization ${ }^{13}$ :

1. There is no alternative drug.

2. The drug involved in DHR is more effective (better quality of life; better survival) or associated with fewer adverse effects than alternative drugs.

3. The drug involved in DHR has a unique mechanism of action, such as aspirin in Aspirin Exacerbated Respiratory Disease (AERD).

Special situations in pediatrics where drug desensitization could be prescribed ${ }^{14}$ :

1. Children suffering from severe and chronic infectious diseases (tuberculosis, Human immunodeficiency virus (HIV) infection, or cystic fibrosis).

2. Children presenting with hematologic or oncologic conditions.

3. Children suffering from chronic inflammatory diseases requiring nonsteroidal anti-inflammatory drugs (NSAIDs) and/or monoclonal antibodies (mAbs).

4. Children allergic to vaccines.

Following are the contraindications of drug desensitization ${ }^{13}$ :

1. Relative to DHR: SCAR (TEN, SJS, DRESS, AGEP), life-threatening immunocytotoxic reactions, and vasculitis are absolute contraindications.

2. Relative to patient: Severe anaphylaxis; uncontrolled asthma (Forced Expiratory Volume (FEV1) < 70\% of their standard value); uncontrolled cardiac diseases; renal or hepatic diseases; use of beta-blockers.

\section{General rules for drug desensitization in children}

The general rules of drug desensitization in adults are also applied to children. Drug desensitization may be used in children reporting DHR primarily to antibiotics, chemotherapies, aspirin, monoclonal antibodies, and vaccines. ${ }^{14,15}$

Physicians and patients (and their caregivers) must be aware that drug desensitization may be associated with a possible risk of acute DHR during the procedure. An informed consent signed by parents and/or tutors is recommended..$^{14,15}$

Drug desensitization protocols are developed to provide the full therapeutic dose of drug without causing severe life-threatening reactions. In any case, this is a highrisk procedure that requires the introduction of potentially lethal medication to a sensitized patient, and therefore the risk-benefit ratio must always be evaluated on an individual basis. ${ }^{16}$

Furthermore, drug desensitization could also be indicated to patients having a history highly suggestive of hypersensitivity reactions but without confirmatory allergic tests. Success of drug desensitization depends on risk stratification and involves analysis of the initial reaction (type, severity, and skin test results) and the presence of prevailing factors in patient such as comorbidities and use of medication. Evaluation starts with a detailed clinical history and some diagnostic tests. Reactions are categorized as follows: mild (only skin symptoms), moderate (skin, respiratory, and gastrointestinal manifestations), and severe (hypoxemia, hypotension, syncope, seizures, cardiac, or respiratory arrest). Serum tryptase measurement at the time of the reaction helps to detect mast cell degranulation (IgE or non-IgE-mediated reactions). ${ }^{16}$ Immediate skin tests could be used to investigate an IgE-mediated mechanism in situations where there is good accuracy, for example, hypersensitivity to beta-lactams and platinums. ${ }^{16,17}$

\section{Aims of desensitization}

Drug desensitization is primarily performed in IgE-mediated reactions and in reactions where drug-specific IgE has not been demonstrated. ${ }^{4,7,13}$ Drug desensitization induces a temporary tolerant state, which can only be maintained by the continuous administration of medication. Two types of DS protocols are available: (i) rapid drug desensitization, which addresses type I reactions with mast cells/basophils/lgE involvement, and (ii) slow drug desensitization, which addresses delayed type IV reactions with T-cells involvement. ${ }^{13}$

\section{Desensitization versus Drug Provocation Test}

It is essential to differentiate between drug desensitization, a therapeutic procedure, and drug provocation test (DPT), a diagnostic test. ${ }^{13}$

Drug provocation test is the controlled administration of drug to diagnose an immune-mediated or nonimmunemediated DHR. It is usually the last step in the diagnostic evaluation if either other tests are negative or unavailable. The purpose of DPT is to confirm or refute hypersensitivity. ${ }^{18,19}$ Drug desensitization aims to induce temporary tolerance to the offending drug..$^{13}$ In both procedures, there are inherent risks. Still, in drug desensitization, there are usually more significant risks because the patient has proven to have highly suspected hypersensitivity, while in DPT, the hypersensitivity is not proven. Sometimes, the DS procedure is used as a precaution even if hypersensitivity is not proven. ${ }^{13}$

There are fewer steps (typically, -three to five) in DPT than more than 10 steps used in drug desensitization (typically, 12 or 16 steps). In DPT, the starting dose is $1 / 100$ or $1 / 10$ of the therapeutic dose. In drug desensitization, the starting dose is much lower, $1 / 100,000$ or $1 / 10,000$ of the therapeutic dose. ${ }^{13,18}$ If a patient has an objective reaction during DPT, the test is stopped, and the patient is treated. During drug desensitization, if the patient presents any clinical manifestations, the administration is interrupted, the patient is treated according to his symptoms, and the procedure is continued after the symptoms are resolved..$^{13,19}$ 


\section{Safety issues in desensitization in children}

Before starting drug desensitization, it is imperative to assess the child with a detailed history. Then perform skin tests or in vitro tests if valuable (good sensitivity and specificity) to characterize the nature of adverse reaction. ${ }^{16,17}$

It is very relevant to study the DS protocol. Preference must be given to protocols already widely used and published in international literature. ${ }^{14,15}$ Occasionally, there may be a need for some changes in protocol according to the child's reactions during drug desensitization.

Drug desensitization is associated with the risk of hypersensitivity reactions, and hence must be performed in a controlled setting under the supervision of a well-trained physician familiar with the procedure and treatment of anaphylaxis. All nurses must be trained to recognize symptoms of hypersensitivity reactions. ${ }^{13-15}$ Equipment and medications to treat allergic reactions and for cardiopulmonary resuscitation must be available. ${ }^{13}$ An intravenous (iv) line and continuous monitoring are required, especially for immediate reactions. An intensive care unit is not obligatory for all children. Many experienced centers do it on an outpatient basis. In any case, for children who have completed the first procedure with few symptoms, subsequent procedures could be performed in the outpatient clinic. ${ }^{13-15}$

There is no consensus on the value of premedication prior to drug desensitization. Possible administrable drugs include $\mathrm{H} 1$ and $\mathrm{H} 2$-antihistamines, corticosteroids, and montelukast, but there are no sufficient data available for recommendations on this topic. ${ }^{14}$

Both oral and parenteral routes could be used as a mode of drug administration as both seem equally effective. The oral route seems to be safer, easier, and less expensive, but blood levels could be affected by gastric $\mathrm{pH}$ and absorption. ${ }^{14}$

In general, the same protocols proposed for the adult population could be applied to children. The only difference with adults is the cumulative dose, which must be the daily dose used for adequate therapy. ${ }^{14}$

In spite of drug desensitization, breakthrough reactions may occur. They most often occur during the first course of drug desensitization. It is mandatory to stop infusion or oral intake in this situation. Most breakthrough reactions require treatment such as antihistamines, corticosteroids, or even adrenaline, but they can resolve just by stopping the infusion, without any additional measures. In such a case, it is important to analyze the best approach for following DS protocols. Further steps or introduction of premedication may be required..$^{13}$ Each patient responds better in a way.

\section{The role of skin tests in desensitization}

Skin tests with drugs are considered safe, with a reported rate of below $1.5 \%$ of systemic reactions in the pediatric population without any fatalities. ${ }^{20}$

Since DS protocols expose sensitized patients to potentially lethal drugs, skin tests help to clarify the mechanisms of reaction in advance, improving the efficacy and safety of the procedure. ${ }^{20}$
Initial testing must be done with higher dilutions and very low titration, with a subsequent increase in titration up to a regular, no irritant concentration test. Skin test must be ideally performed 4 to 6 weeks after the reaction to avoid false-negative results. ${ }^{21}$

Skin tests have a relatively narrow role in diagnosing chemotherapeutic hypersensitivity reactions, since the sensitivity and specificity of the tests are not well established. Nonetheless, literature demonstrates that a negative carboplatin skin test seems to predict with reasonable reliability the absence of severe hypersensitivity reaction during a subsequent infusion of drug. At the same time, a positive result might correlate to a higher risk of reaction during the procedure. Patients may convert from a negative skin test to a positive one after first desensitization. ${ }^{22}$

Protocols with skin tests for platinum hypersensitivity have evidenced that gradual tolerance could be induced in most skin test-negative patients who receive repeated desensitization. Otani et al. developed a strategy to safely advance from an intermediate desensitization protocol to a $50 \%$ standard infusion rate according to the risk stratification of the patient with repeated and subsequent skin tests. ${ }^{22}$

Skin tests can also help identify cross-reaction between platinum compounds, such as carboplatin and oxaliplatin, aiding to recognize the patient with a higher likelihood of tolerating a different platinum agent without desensitization. ${ }^{22}$

Another benefit of skin testing before desensitizing is to recognize the best starting dose for a desensitization protocol, as the initial concentration could be determined based on the endpoint titration in the test. ${ }^{21}$

Skin tests have a less established role in the evaluation of taxane hypersensitivity reactions, since most reactions are thought to be non-lgE-mediated. However, a subset of patients might be previously sensitized to taxanes through an IgE-mediated mechanism based on cross-reactivity between taxanes and pollen from yew trees. ${ }^{23,24}$

Otani et al. have recently proposed a risk stratification strategy performing skin tests in addition to grading the severity of initial reaction to determine whether it is safe to reintroduce platinum or taxane agents through standard infusion. ${ }^{23,25}$

Patients with more severe reactions to taxanes are more likely to have a positive skin test results. ${ }^{23}$

\section{The rapid desensitization protocols}

\section{General considerations}

Drug desensitization is mostly unfamiliar and unnoted by pediatric oncologists. However, it is a great strategy to avoid premature treatment cessation, allowing patients to keep treatment with first-line agents. Less literature is available on desensitization in children, and most pediatric protocols have been adapted from adult ones. ${ }^{14}$

The standard desensitization protocol aims to block mast cells' release of preformed mediators, cytokine production, and calcium influx, impending degranulation of mast cells if exposed to a specific drug. However, the exact 
mechanism of desensitization has not been fully elucidated yet. Some suspect a longer memory of tolerance induced by desensitization, with modifications of immune response and increased regulatory cytokines such as $\mathrm{IL}-10$ and $\mathrm{T}$ regulatory cells during desensitization. ${ }^{26}$

The most widely accepted protocol consists of a 12-step three-bag model, in which antigen doses are offered cumulatively, being doubled every $15 \mathrm{~min}$, starting at a maximum concentration of $1 / 100$ of the final dose. Three bags are administered consecutively; the first bag contains a $1 / 100$ dilution, the second one a 1/10 dilution, and the third one is calculated by subtracting the total dose administered in the first two bags from the total target dose. Tolerance is usually reached in $6 \mathrm{~h}$, and it lasts for only three to four half-lives of the drug (Table 1). The state of tolerance usually endures while the patient is receiving the drug. When administration stops, tolerance is lost, requiring a new desensitization protocol for each treatment cycle. ${ }^{27}$

Depending on the risk stratification, different protocols with a different number of bags, steps, and dilutions could be used. High-risk patients and patients with severe reactions or breakthrough reactions during a 12-step three-bag protocol can benefit from a 16-step four-bag protocol. Similarly, patients with low risk, initial negative skin test results, and mild reactions can tolerate an 8 -step two-bag protocol. ${ }^{24}$

Drug desensitization is restricted to specific scenarios and must be considered after a cautious positive riskbenefit analysis. The procedure must take place preferably in a hospital setting under close monitoring and supervision by trained personnel. ${ }^{27}$

The best candidates for drug desensitization are patients with immediate IgE-mediated drug allergies. However, other mechanisms of reaction such as cytokine storm-like reactions, mixed reactions, or even non-severe delayed type IV reactions might be treated with desensitization. ${ }^{28}$
Drug desensitization is contraindicated in patients with a clinical history of SCAR, such as TEN, SJS, or AGEP. Type II or III mechanisms such as serum sickness disease or hemolytic anemia are also not indicated for desensitization. ${ }^{14}$

\section{Pretreatment}

There is no consensus about premedication before desensitization. However, it is a common practice. Premedication protocols vary from one center to another and help to prevent or minimize the severity of allergic reactions. Premedication does not avoid anaphylactic reactions, so epinephrine and cardiopulmonary resuscitation (CPR) equipment must always be available in the setting of the procedure to treat a possible anaphylactic reaction. ${ }^{29}$

Premedication is usually individualized and targeted at patient's symptoms. Montelukast is usually added as a premedication if the patient experiences flushing; glucocorticoids and bronchodilators are added for bronchospasm, and acetaminophen or nonsteroidal anti-inflammatory drugs are kept to manage chills, pain, or fever. Nonsedating antihistamines, up to a conventional dose of four times (QID), are a good strategy for urticarial symptoms. Other premedication includes intravenous fluids, $\mathrm{H} 2$ blockers (H2-receptor antagonists), doxepin, ondansetron, gabapentin, and lorazepam, used according to requirement or patient preference. ${ }^{28}$

Literature and daily clinical practice demonstrate that hypersensitivity reactions frequently occur during the final bag infusion, given the significant amount of antigen contained in the last bag. The second stage of premedication might be helpful just before initiating the last bag, with good results preventing reactions. ${ }^{28}$

Drug interactions and adverse effects, such as hyperglycemia and immunosuppression with corticosteroids,

Table 1 The 12-step rapid desensitization protocol adapted for laronidase. ${ }^{36}$

\begin{tabular}{|c|c|c|c|c|c|c|}
\hline & \multicolumn{2}{|r|}{ Volume (mL) } & \multicolumn{2}{|c|}{ Concentration $(\mathrm{mg} / \mathrm{mL})$} & \multicolumn{2}{|c|}{$\begin{array}{c}\text { Total amount of drug in each } \\
\text { solution }(\mathrm{mg})\end{array}$} \\
\hline Solution 1 & \multicolumn{2}{|r|}{250} & \multicolumn{2}{|r|}{0.000812} & \multicolumn{2}{|c|}{0.2030} \\
\hline Solution 2 & & 250 & & 0.00812 & \multicolumn{2}{|c|}{2.0300} \\
\hline Solution 3 & & 250 & & 0.08056 & 20. & 403 \\
\hline Step No. & Solution No. & Rate (mL/h) & Time (min) & $\begin{array}{l}\text { Volume infused } \\
\text { per step (mL) }\end{array}$ & $\begin{array}{l}\text { Administered dose } \\
\quad(\mathrm{mg})\end{array}$ & $\begin{array}{l}\text { Cumulative } \\
\text { dose (mg) }\end{array}$ \\
\hline 1 & 1 & 2.0 & 15 & 0.50 & 0.0004 & 0.0004 \\
\hline 2 & 1 & 5.0 & 15 & 1.25 & 0.0010 & 0.0014 \\
\hline 3 & 1 & 10.0 & 15 & 2.50 & 0.0020 & 0.0035 \\
\hline 4 & 1 & 20.0 & 15 & 5.00 & 0.0041 & 0.0075 \\
\hline 5 & 2 & 5.0 & 15 & 1.25 & 0.0102 & 0.0177 \\
\hline 6 & 2 & 10.0 & 15 & 2.50 & 0.0203 & 0.0380 \\
\hline 7 & 2 & 20.0 & 15 & 5.00 & 0.0406 & 0.0786 \\
\hline 8 & 2 & 40.0 & 15 & 10.00 & 0.0812 & 0.1598 \\
\hline 9 & 3 & 10.0 & 15 & 2.50 & 0.2014 & 0.3612 \\
\hline 10 & 3 & 20.0 & 15 & 5.00 & 0.4028 & 0.7640 \\
\hline 11 & 3 & 40.0 & 15 & 10.00 & 0.8056 & 1.5696 \\
\hline 12 & 3 & 80.0 & 174.375 & 232.50 & 18.7305 & 20.300 \\
\hline
\end{tabular}

Total time $=340 \mathrm{~min}(5.6 \mathrm{~h})$. 
drowsiness, and electrocardiogram (ECG)-prolonged QT interval with first-generation antihistamines, have been observed. Therefore, unnecessary premedication must be avoided, and clinical indications must be individualized and reviewed before every new procedure. ${ }^{29}$

\section{Treating reactions during desensitization}

If the patient experiences a mild reaction during drug desensitization, the infusion should be paused, and medications must be administered based on symptoms. After managing the symptoms, the infusion can be resumed from the point where it was stopped. ${ }^{30}$

However, if a severe reaction develops, desensitization must be stopped promptly and the patient treated appropriately. Infusion should not be resumed, and the patient must stay under close observation and monitoring for delayed reactions. The best practice is to reevaluate the whole desensitization protocol, adjust premedication, and increase the number of steps for the future infusion. ${ }^{30}$

First-line treatment for anaphylaxis includes intramuscular adrenaline before any other intervention. Adrenaline must be injected intramuscularly at the mid-outer thigh at a dose of $0.01 \mathrm{~mL} / \mathrm{kg}$ of aqueous adrenaline $1: 1000$ (up to $0.5 \mathrm{~mL}$ ) and if required, can be repeated every 5 to $15 \mathrm{~min}$. If the patient's weight is unknown, an approximate dosage is $50 \mu \mathrm{g}$ for infants aged less than 6 months; $120 \mu \mathrm{g}$ for children aged between 6 months and 6 years; $250 \mu \mathrm{g}$ from 6 to 12 -year-old children, and $500 \mu \mathrm{g}$ for children older than 12 years. ${ }^{31}$

In addition, the patient should be positioned appropriately, and high-flow oxygen must be administered if the child presents with desaturation. The child must be kept supine, with raised legs, to promote the perfusion of vital organs, in Trendelenburg position if possible. ${ }^{31}$

For patients with cardiovascular instability, fluid support must be initiated, and inhaled short-acting beta-2 agonists could be given to relieve bronchoconstriction symptoms. $\mathrm{H} 1$ antihistamines and $\mathrm{H} 2$ blockers act as a second-line of therapy for cutaneous symptoms. Glucocorticoids help to prevent prolonged anaphylactic symptoms or biphasic anaphylaxis. ${ }^{31}$

Up to $15 \%$ of the children develop a biphasic reaction after complete recovery of anaphylaxis with no further exposure to allergen. So, it is highly recommended to observe the patient and monitor vital signs at frequent and regular intervals for at least $6-8 \mathrm{~h}$ in case of respiratory compromise and at least $12-24 \mathrm{~h}$ for patients who presented with hypotension. The duration of the observation must be individualized and guided by the severity of initial reaction, response to treatment, individual comorbidities, and half-life of the drug implicated in the reaction. ${ }^{30}$

\section{Chemotherapy}

Carboplatin and L-asparaginase are the two primary chemotherapeutic agents associated with DHR in pediatrics. Hypersensitivity reactions to carboplatin are mostly described in patients with low-grade glioma, and up to $47 \%$ of these patients react to carboplatin. The incidence of carboplatin hypersensitivity reaction in solid tumors is around $9 \%$ in the pediatric population. L-asparaginase hypersensitivity reactions occur in about $40 \%$ of the children affected by lymphoblastic leukemia. ${ }^{32,33}$

A primary difference while structuring a DS protocol for a chemotherapeutic agent compared to other drugs is that the chemotherapy dose is calculated per square meter of body surface per day. The full therapeutic dose differs from child to child, and protocols must be tailored for each patient and modified if the patient gains weight. ${ }^{32}$

L-asparaginase is a highly reactive molecule because of its high molecular weight and complex structure, which leads to formation of asparaginase-specific antibody. The measurement of these types of antibodies might help to predict future hypersensitivity to the drug. ${ }^{33}$

The initial hypersensitivity reaction to a platinum agent occurs typically after multiple cycles, usually around the eighth or ninth course overall, after a period of possible IgE sensitization. Reactions are likely to be mast cell-mediated, although the exact mechanism remains unclear. The most widely accepted desensitization protocols for platinum agents are the 8 -step and 12-step protocols. ${ }^{22}$

Platinum agents can induce type I reactions, cytokine-release reactions, or mixed ones. Most reactions develop during the infusion or within hours of infusion. Oxaliplatin hypersensitivity reactions are more heterogeneous and might present with atypical symptoms such as fevers, chills, rigor, chest or back pain, and elevated levels of IL- 6 and TNF- $\alpha .{ }^{24}$

Taxane hypersensitivity reactions often occur on the first or second exposure. These usually occur within minutes after the beginning of the infusion. Taxanes may cause mast cell or direct basophil activation, activation through IgE-mediated mechanism, or IgG-mediated mechanism. IgG immune complexes can activate pathways of the complementary system resulting in the generation of anaphylatoxins ( $\mathrm{C} 3 \mathrm{a}$ and $\mathrm{C} 5 \mathrm{a})$. Solvents associated with taxanes, such as cremophor EL and polysorbate 80, may also activate mast cells through IgE-mediated mechanism or activation of complement pathways. Desensitization is not indicated in patients having direct mast cell or basophil activation. ${ }^{24}$

The number of reactions during drug desensitization to chemotherapy or monoclonal antibodies gradually reduces with the number of desensitizations. Meanwhile, the percentage of patients who tolerate the desensitization procedure during repeated procedure cycles increases over time. ${ }^{22,28}$

\section{Biologics}

Hypersensitivity reactions to biologics in children have been increasingly documented, primarily because of the rise in its use, as clinical applications to biologics become broader. ${ }^{28}$ Hypersensitivity reactions can occur after multiple exposures or at the first one, as seen with cetuximab and patients with IgE antibodies against galactose- $\alpha-1,3$-galactose. ${ }^{34}$

The clinical presentation of hypersensitivity reactions to biologics may differ accordingly to the agent involved, the mechanism of reaction, or the patient. An overlap 
of several mechanisms has been described, and diagnosis and management protocols are yet to be standardized. Reactions may be infusion-related, cytokine-release reactions, type I (IgE/non-IgE), type III, and delayed type IV reactions. Severity ranges from mild symptoms to life-threatening situations. ${ }^{35}$

Infusion reactions and cytokine-release reactions to biologics can occur at the first exposure and present with flushing, chills, fever, tachycardia, hypertension, dyspnea, nausea, vomiting, and syncope. An easy way to differentiate one from another is that infusion reactions are usually self-limited, symptoms are usually mild to moderate, and respond very well to premedication and adjustments in the infusion rate. Infusion-related reactions do not require desensitization, while cytokine-release reactions may improve and be treated with it. ${ }^{28}$

Type I reactions manifest with pruritus, flushing, angioedema, urticaria, dyspnea, hypotension, gastrointestinal symptoms, or even anaphylaxis, and are a classic indication for desensitization. Type III reactions can be local, such as Arthus reaction, or systemic such as serum-like disease, and have been described with infliximab, etanercept, and adalimumab. Desensitization appears not to be useful in this type of reaction. ${ }^{35}$

Delayed type IV reactions vary from delayed maculopapular rashes to severe reactions such as SJS, appearing at $12 \mathrm{~h}$ to several weeks after the exposure. ${ }^{35}$

Subsequent reactions to biologics during desensitization are usually milder than initial ones. The most common initial symptoms are cutaneous, followed by respiratory symptoms. On the other hand, during desensitization, the predominant symptoms are related to cytokine-release reactions. The mechanism of reaction might change from an initial reaction to a subsequent reaction during desensitization. ${ }^{35}$

Interestingly, patients receiving prophylactic intravenous fluids as premedication had less severe grading reactions, while the patients that received intravenous fluids just after the breakthrough reaction had a decrease in their severity. According to the authors, intravenous fluids would dilute both antigens and mast-cell mediators, decreasing their concentration and allowing a better clearance. ${ }^{28}$

Lysosomal diseases represent a heterogeneous group of rare disorders with chronic progression and functional impairment of different organs that can be delayed with enzyme replacement therapy (ERT). As with other biologics, ERT may induce hypersensitivity reactions that make continuation of treatment impossible. Desensitization has been successfully described in laronidase (type-I mucopolyssacharidosis [MPS-I]), arylsulfatase B (MPS-VI), galsufase (MPS-VI), aglucosidase alfa (Pompe disease), and sebelipase alfa (lysosomal acid lipase deficiency) (Table 1). ${ }^{36-41}$

\section{Other protocols}

\section{Antibiotics}

Antibiotics are the first cause of DHR in children in the United States and the second cause in Latin America. ${ }^{42,43}$ However, allergy can be excluded in most patients after a complete workup, and effective alternative antibacterial therapy is available most of the time. A specific situation in which the patient may have no therapeutic options other than the antibiotic causing hypersensitivity is cystic fibrosis. ${ }^{44}$

Turvey et al. described a protocol starting at $1 / 1,000,000$ of the full therapeutic dose delivered by continuous intravenous infusion for $30 \mathrm{~min}$ and proceeding with 10 -fold dose increase until reaching the total therapeutic dose. ${ }^{44}$ Their protocol was used to perform 57 desensitizations in 21 adults and children (19 with cystic fibrosis) with a suggestive history of an IgE-mediated reaction to an antibiotic and a positive skin test in most of them, with a successful outcome in $75 \%$ cases. A review of the patients where the desensitization failed suggests that in 7 of the 11 patients, the reaction mechanism was not IgE-mediated. ${ }^{44}$

Legere et al. reported using the 12-step rapid desensitization protocol in 15 adult patients with cystic fibrosis. ${ }^{45}$ Fifty of the 52 desensitizations (96.2\%) were completed without adverse events. The high success rate of this protocol could be explained by the exclusion of patients with no typical type I hypersensitivity reactions and adapting the protocol when necessary. ${ }^{45}$

Trimethoprim-sulfamethoxazole (TMP-SMX) is frequently used in prophylaxis against Pneumocystis carinii pneumonia in children with different conditions. ${ }^{46-49}$ The most frequent manifestations of TMP-SMX hypersensitivity are due to T-cell-mediated reactions, but IgE-mediated urticaria and anaphylaxis may also take place. ${ }^{50}$ Different protocols have been proposed for TMP-SMX desensitization, including a 4-h rapid oral protocol for children infected with HIV, a 5-day oral protocol for chronic granulomatous disease, and a 17-day protocol for allogeneic hematopoietic stem cell transplantation. ${ }^{46,48,49}$ Although less frequent, desensitization to non-immediate TMP-SMX hypersensitivity reaction may also be necessary. ${ }^{51}$

In general, desensitization with antibiotics can be performed through oral, intravenous, or subcutaneous routes, starting with dilutions of 1:100 or 1:1000 of the total therapeutic doses and doubling the dose every 15 to 60 min until reaching the final dose. ${ }^{52}$ Depending on the antibiotic and severity of the reaction, initial dilutions of $1: 1,000,000$ to 1:100,000 are recommended..$^{15}$ After desensitization, antibiotic administration must be maintained for the intended duration. New courses of antibiotics require further desensitization. ${ }^{52}$ Currently, numerous desensitization protocols to antibiotics have been described in adults, including for non-immediate reactions, but there is a lack of data in case of children. As a rule, the primary difference between the desensitization protocols of adults and children is the final cumulative dose. ${ }^{15}$ However, differences in children's metabolism may require some adjustment.

\section{Nonsteroidal anti-inflammatory drugs}

Non-steroidal anti-inflammatory drugs are used worldwide. During childhood and adolescence, a growing number of NSAIDs are available for these age groups. ${ }^{53}$ NSAIDs are an important cause of hypersensitivity reactions and are involved in up to $70 \%$ of drug-induced anaphylaxis 
in children. ${ }^{54}$ Hypersensitivity to NSAIDs involves different mechanisms and clinical presentations but urticaria/ angioedema are the most common manifestations. These reactions can be IgE-mediated (selective hypersensitivity) or, possibly, the result of cyclooxygenase (COX) inhibition (nonselective hypersensitivity). Avoiding a specific drug and those with a similar structure is recommended in the first case, and all NSAIDs but those weak or selective/specific COX-2 inhibitors in the second, always after a complete workout that involves clinical history, skin, and provocation tests. It is important to emphasize that we can exclude drug hypersensitivity with a complete investigation in most cases. ${ }^{53}$

Current literature has no reports on desensitization to NSAIDs in the pediatric age group. Classic indications for aspirin desensitization in adults include NSAID-exacerbated respiratory disease (NERD) and nasal polyposis and prophylaxis or treatment of coronary disease. However, requirement to perform desensitization for these conditions in children is uncommon. An age/weight/disease-adjusted established protocol used in adults is suggested if NSAIDs desensitization is indicated in children. ${ }^{53}$

\section{Vaccines}

Billions of vaccine doses are administered worldwide every year for the prevention of infectious diseases. Adverse events to vaccines are uncommon, but the occurrence of an adverse reaction may result in incorrect labeling of a child as allergic. ${ }^{55}$ The European Anaphylaxis Registry (NORA) reported that $0.6 \%$ of all drug-induced anaphylaxis is caused by vaccines. ${ }^{56}$ Potential allergens contained in vaccines include gelatin and Hen's egg proteins. Moreover, adjuvants such as aluminum, thimerosal, phenoxyethanol, and formaldehyde may also be related to hypersensitivity reactions to vaccines. Latex, antibiotics, yeast, milk, and dextran are other potential allergenic vaccine components. ${ }^{55,57}$ With the COVID-19 pandemic, new vaccines have been developed and millions of people are vaccinated around the world. Consequently, hypersensitivity reactions, including anaphylaxis, have appeared, and polyethylene glycol (PEG) and polysorbate 80 are reported as possible causes as well. ${ }^{56,57}$

Investigation of vaccine's hypersensitivity reactions must be tailor-made, according to the vaccine and type of reaction, and include the analysis of plasma lgE markers, basophil and mast cells activation tests, and skin tests. ${ }^{57,58}$ As the diagnosis of allergy is confirmed to any of the vaccine's components, a risk assessment for anaphylaxis is critical as well as for not taking the vaccine.

Few reports have appeared of desensitization to vaccines. ${ }^{15}$ In general, vaccine can be administered in a usual manner to patients with negative skin tests because an IgEmediated allergy is unlikely to take place. An alternative vaccine not containing the implicated component is indicated for patients with positive skin tests and a compatible history of an IgE-mediated reaction. If a patient requires an additional dose or any other vaccine that contains the incriminated component, then its administration must be fractioned according to specific protocols. ${ }^{55}$

\section{Future Perspective}

Increasing number of new drugs are available in the market every year, especially biologics and small molecules. Thus, in the near future, we are probably facing hypersensitivity reactions to these drugs as well. Therefore, standardized tests for an accurate diagnosis and desensitization protocols are required, including specific ones for the pediatric population.

\section{Conclusions}

Drug desensitization enables children allergic to drugs to receive their treatment in a safe manner, especially when the offending drug is the treatment of choice and no other safe and effective medication is available. Examples include patients with cancer that had an allergic reaction to the first-line chemotherapeutic agent, children with cystic fibrosis with antibiotic hypersensitivity, or those who require a biologic previously involved in a DHR to control their disease. Many desensitization protocols are described in literature but not for children. The 12-step rapid desensitization protocol is a safe and effective protocol that could be adapted to almost all injectable drugs in the pediatric group.

\section{References}

1. Muraro A, Lemanske RF, Jr, Castells M, Torres MJ, Khan D, Simon HU, et al. Precision medicine in allergic disease-food allergy, drug allergy, and anaphylaxis-PRACTALL document of the European Academy of Allergy and Clinical Immunology and the American Academy of Allergy, Asthma and Immunology. Allergy. 2017;72(7):1006-21. http://dx.doi. org/10.1111/all.13132

2. Demoly P, Adkinson NF, Brockow K, Castells M, Chiriac AM, Greenberger PA, et al. International consensus on drug allergy. Allergy. 2014;69(4):420-37. http://dx.doi.org/10.1111/ all.12350

3. Pichler WJ. Delayed drug hypersensitivity reactions. Ann Intern Med. 2003;139(8):683-93. http://dx.doi.org/10.7326/0003-4819139-8-200310210-00012

4. de Las Vecillas Sánchez L, Alenazy LA, Garcia-Neuer M, Castells MC. Drug hypersensitivity and desensitizations: Mechanisms and new approaches. Int J Mol Sci. 2017;18(6):1316. http://dx.doi.org/10.3390/ijms18061316

5. Picard M, Pur L, Caiado J, Giavina-Bianchi P, Galvão VR, Berlin ST, et al. Risk stratification and skin testing to guide re-exposure in taxane-induced hypersensitivity reactions. J Allergy Clin Immunol. 2016;137(4):1154-64.e12. http://dx.doi. org/10.1016/j.jaci.2015.10.039

6. Giavina-Bianchi P, Galvão VR, Picard M, Caiado J, Castells MC. Basophil activation test is a relevant biomarker of the outcome of rapid desensitization in platinum compounds allergy. J Allergy Clin Immunol Pract. 2017;5(3):728-36. http://dx.doi. org/10.1016/j.jaip.2016.11.006

7. Sloane D, Govindarajulu U, Harrow-Mortelliti J, Barry W, Hsu FI, Hong D, et al. Safety, costs, and efficacy of rapid drug desensitizations to chemotherapy and monoclonal antibodies. J Allergy Clin Immunol Pract. 2016;4(3):497-504. http://dx.doi. org/10.1016/j.jaip.2015.12.019 
8. Ang WX, Church AM, Kulis M, Choi HW, Burks AW, Abraham SN. Mast cell desensitization inhibits calcium flux and aberrantly remodels actin. J Clin Invest. 2016;126(11):4103-18. http://dx. doi.org/10.1172/JCl87492

9. McNeil BD, Pundir P, Meeker S, Han L, Undem BJ, Kulka M, Dong $X$. Identification of a mast-cell-specific receptor crucial for pseudo-allergic drug reactions. Nature. 2015;519(7542):237-41. http://dx.doi.org/10.1038/nature14022

10. Sancho-Serra Mdel C, Simarro M, Castells M. Rapid IgE desensitization is antigen specific and impairs early and late mast cell responses targeting Fc\&RI internalization. Eur J Immunol. 2011;41(4):1004-13. http://dx.doi.org/10.1002/eji.201040810

11. Oka T, Rios EJ, Tsai M, Kalesnikoff J, Galli SJ. Rapid desensitization induces internalization of antigen-specific lgE on mouse mast cells. J Allergy Clin Immunol. 2013;132(4):922-32. e1-16. http://dx.doi.org/10.1016/j.jaci.2013.05.004

12. Shalit M, Levi-Schaffer F. Challenge of mast cells with increasing amounts of antigen induces desensitization. Clin Exp Allergy. 1995;25(9):896-902. http://dx.doi.org/10.1111/j.1365-2222.1995. tb00033.x

13. Cernadas JR, Brockow K, Romano A, Aberer W, Torres MJ, Bircher A, et al; European network of drug allergy and the $\mathrm{EAACl}$ interest group on drug hypersensitivity. General considerations on rapid desensitization for drug hypersensitivity-A consensus statement. Allergy. 2010;65(11):1357-66. http://dx. doi.org/10.1111/j.1398-9995.2010.02441.x

14. Diaferio L, Giovannini M, Clark E, Castagnoli R, Caimmi D. Protocols for drug allergy desensitization in children. Expert Rev Clin Immunol. 2020;16(1):91-100. http://dx.doi.org/10.108 0/1744666X.2019.1698294.

15. Caimmi S, Caffarelli C, Saretta F, Liotti L, Crisafulli G, Cardinale $\mathrm{F}$, et al. Drug desensitization in allergic children. Acta Biomed. 2019;90(3-S):20-9. http://dx.doi.org/10.23750/ abm.v90i3-S.8158

16. Castells Guitart MC. Rapid drug desensitization for hypersensitivity reactions to chemotherapy and monoclonal antibodies in the 21st century. J Investig Allergol Clin Immunol. 2014;24(2):72-9. PMID: 24834769

17. Hong DI, Dioun AF. Indications, protocols, and outcomes of drug desensitizations for chemotherapy and monoclonal antibodies in adults and children. J Allergy Clin Immunol Pract. 2014;2(1):139; quiz 20. http://dx.doi.org/10.1016/j.jaip.2013.11.007

18. Macy E, Ensina LF. Is skin testing required prior to drug challenges? J Allergy Clin Immunol Pract. 2018;7(2):412-7.

19. Soyer O, Sahiner UM, Sekerel BE. Pro and contra: Provocation tests in drug hypersensitivity. Int J Mol Sci. 2017;18(7):1437. http://dx.doi.org/10.3390/ijms18071437

20. Gomes ER, Brockow K, Kuyucu S, Saretta F, Mori F, BlancaLopez N, et al. Drug hypersensitivity in children: Report from the pediatric task force of the EAACI Drug Allergy Interest Group. Allergy. 2016 Feb;71(2):149-61. http://dx.doi. org/10.1111/all.12774

21. Brockow K, Romano A, Blanca M, Ring J, Pichler W, Demoly P. General considerations for skin test procedures in the diagnosis of drug hypersensitivity. Allergy. 2002 Jan;57(1):45-51. PMID: 11991289

22. Otani IM, Wong J, Banerji A. Platinum chemotherapy hypersensitivity: Prevalence and management. Immunol Allergy Clin North Am. 2017 Nov;37(4):663-77. http://dx.doi.org/10.1016/j. iac.2017.06.003

23. Otani IM, Lax T, Long AA, Slawski BR, Camargo CA, Jr, Banerji A. Utility of risk stratification for paclitaxel hypersensitivity reactions. J Allergy Clin Immunol Pract. 2018 JulAug;6(4):1266-73.e2. http://dx.doi.org/10.1016/j.jaip.2017.08 025

24. Tsao LR, Young FD, Otani IM, Castells MC. Hypersensitivity reactions to platinum agents and taxanes. Clin Rev
Allergy Immunol. 2021 Aug 2. http://dx.doi.org/10.1007/ s12016-021-08877-y

25. Otani IM, Castells $M$. Novel roles for platinum and taxane agent skin testing in risk stratification of chemotherapy hypersensitivity. J Allergy Clin Immunol Pract. 2020 May;8(5):166567. http://dx.doi.org/10.1016/j.jaip.2020.02.008

26. Vultaggio A, Matucci A, Nencini F, Bormioli S, Vivarelli E, Maggi E. Mechanisms of drug desensitization: Not only mast cells. Front Pharmacol. 2020 Dec 23;11:590991. http://dx.doi. org/10.3389/fphar.2020.590991

27. Castells M. Rapid desensitization for hypersensitivity reactions to medications. Immunol Allergy Clin North Am. 2009 Aug;29(3):585-606. http://dx.doi.org/10.1016/j.iac.2009.04.012.

28. Isabwe GAC, Garcia Neuer M, de Las Vecillas Sanchez L, Lynch DM, Marquis K, Castells M. Hypersensitivity reactions to therapeutic monoclonal antibodies: Phenotypes and endotypes. J Allergy Clin Immunol. 2018 Jul;142(1):159-170.e2. http://dx.doi.org/10.1016/j.jaci.2018.02.018

29. Lopez-Gonzalez P, Madrigal-Burgaleta R, Carpio-Escalona LV, Bernal-Rubio L, Guerra E, Berges-Gimeno MP, et al. Assessment of antihistamines and corticosteroids as premedication in rapid drug desensitization to Paclitaxel: Outcomes in 155 procedures. J Allergy Clin Immunol Pract. 2018 Jul-Aug;6(4):1356-62. http://dx.doi.org/10.1016/j.jaip. 2017.11.013

30. Atanaskovic-Markovic M, Gomes E, Cernadas JR, du Toit G, Kidon $M$, Kuyucu $S$, et al. Diagnosis and management of drug-induced anaphylaxis in children: An EAACl position paper. Pediatr Allergy Immunol. 2019 May;30(3):269-76. http://dx.doi.org/10.1111/pai.13034

31. Martelli A, Ippolito R, Votto M, De Filippo M, Brambilla I, Calvani $M$, et al. What is new in anaphylaxis? Acta Biomed. 2020 Sep 15;91(11-S):e2020005. http://dx.doi.org/10.23750/ abm.v91i11-S.10308

32. Lafay-Cousin L, Sung L, Carret AS, Hukin J, Wilson B, Johnston DL, et al. Carboplatin hypersensitivity reaction in pediatric patients with low-grade glioma: A Canadian Pediatric Brain Tumor Consortium experience. Cancer. 2008 Feb 15;112(4):892-9. http://dx.doi.org/10.1002/cncr.23249

33. Akbayram S, Doğan M, Akgün C, Caksen H, Oner AF. A desensitization protocol in children with L-asparaginase hypersensitivity. J Pediatr Hematol Oncol. 2010 Jul;32(5):e187-91. http://dx.doi.org/10.1097/MPH.0b013e3181e003c7

34. Chung $\mathrm{CH}$, Mirakhur B, Chan E, Le QT, Berlin J, Morse M, et al. Cetuximab-induced anaphylaxis and IgE specific for galactose-alpha-1,3-galactose. N Engl J Med. 2008 Mar 13;358(11):1109-17. http://dx.doi.org/10.1056/NEJMoa074943

35. Hong DI, Bankova L, Cahill KN, Kyin T, Castells MC. Allergy to monoclonal antibodies: Cutting-edge desensitization methods for cutting-edge therapies. Expert Rev Clin Immunol. 2012 Jan;8(1):43-52; quiz 53-4. http://dx.doi.org/10.1586/eci.11.75

36. Ensina LF, Aranda CS, de Lacerda AE, Camelo-Nunes I, Sole D, Martins AM, et al. Laronidase hypersensitivity and desensitization in type I mucopolysaccharidosis: A case report. Pediatr Allergy Immunol. 2014;25(5):498-9. http://dx.doi.org/10.1111/ pai.12209

37. Aranda CS, Ensina LF, Nunes IC, Mallozi MC, Mendes C, Martins AM, et al. Diagnosis and management of infusion-related hypersensitivity reactions to enzyme replacement therapy for lysosomal diseases: The role of desensitization. J Allergy Clin Immunol Pract. 2016;4(2):354-6. http://dx.doi. org/10.1016/j.jaip.2015.11.012

38. Bekis Bozkurt H, Karakurt T, Cavkaytar O, Arga M. Shortened desensitization leading to a 2-year enzyme replacement therapy with elosulfase alfa. Ann Allergy Asthma Immunol. 2021;127(2):261-2. http://dx.doi.org/10.1016/j.anai.2021. 04.040 
39. Toh TSW, Chong KW, Goh AEN, Goh JCY, Ting TW, Tan ES, et al. Enzyme replacement therapy desensitization in a child with infantile onset Pompe disease. Asian Pac J Allergy Immunol. 2020. http://dx.doi.org/10.12932/AP-060919-0638

40. Huffaker MF, Liu AY, Enns GM, Vijay S, Amor AJ, Adkinson NF, Jr. Case series of sebelipase alfa hypersensitivity reactions and successful sebelipase alfa rapid desensitization. JIMD Rep. 2019;49(1):30-6. http://dx.doi.org/10.1002/jmd2.12066

41. Capanoglu M, Dibek Misirlioglu E, Azkur D, Vezir E, Guvenir $\mathrm{H}$, Gunduz $M$, et al. IgE-mediated hypersensitivity and desensitisation with recombinant enzymes in Pompe disease and Type I and Type VI mucopolysaccharidosis. Int Arch Allergy Immunol. 2016;169(3):198-202. http://dx.doi.org/10.1159/ 000446154

42. Norton AE, Broyles AD. Management of children with hypersensitivity to antibiotics and monoclonal antibodies. Immunol Allergy Clin North Am. 2017;37(4):713-25. http://dx.doi. org/10.1016/j.iac.2017.07.005

43. Jares EJ, Sánchez-Borges M, Cardona-Villa R, Ensina LF, Arias-Cruz A, Gómez $M$, et al. Multinational experience with hypersensitivity drug reactions in Latin America. Ann Allergy Asthma Immunol. 2014;113(3):282-9. http://dx.doi. org $/ 10.1016 /$ j.anai.2014.06.019

44. Turvey SE, Cronin B, Arnold AD, Dioun AF. Antibiotic desensitization for the allergic patient: 5 Years of experience and practice. Ann Allergy Asthma Immunol. 2004;92(4):426-32. http://dx.doi.org/10.1016/S1081-1206(10)61778-4

45. Legere HJ, 3rd, Palis RI, Rodriguez Bouza T, Uluer AZ, Castells MC. A safe protocol for rapid desensitization in patients with cystic fibrosis and antibiotic hypersensitivity. J Cyst Fibros. 2009;8(6):418-24. http://dx.doi.org/10.1016/j.jcf.2009.08.002

46. Palusci VJ, Kaul A, Lawrence RM, Haines KA, Kwittken PL. Rapid oral desensitization to trimethoprim-sulfamethoxazole in infants and children. Pediatr Infect Dis J. 1996;15(5):45660. http://dx.doi.org/10.1097/00006454-199605000-00015

47. Gómez-Traseira C, Boyano-Martínez T, Escosa-García L, Pedrosa M, Martín-Muñoz F, Quirce S. Trimethoprimsulfamethoxazole (cotrimoxazole) desensitization in an HIV-infected 5-yr-old girl. Pediatr Allergy Immunol. 2015;26(3):287-9. http://dx.doi.org/10.1111/pai.12353

48. Hasui M, Kotera F, Tsuji S, Yamamoto A, Taniuchi S, Fujikawa $Y$, et al. Successful resumption of trimethoprimsulfamethoxazole after oral desensitisation in patients with chronic granulomatous disease. Eur J Pediatr. 2002;161(6):3567. http://dx.doi.org/10.1007/s00431-002-0944-6
49. Soffritti S, Ricci G, Prete A, Rondelli R, Menna G, Pession A. Successful desensitization to trimethoprim-sulfamethoxazole after allogeneic haematopoietic stem cell transplantation: Preliminary observations. Med Pediatr Oncol. 2003;40(4):2712. http://dx.doi.org/10.1002/mpo.10196

50. Sánchez-Borges M, Thong B, Blanca M, Ensina LF, GonzálezDíaz S, Greenberger PA, et al. Hypersensitivity reactions to non beta-lactam antimicrobial agents, a statement of the WAO special committee on drug allergy. World Allergy Organ J. 2013;6(1):18. http://dx.doi.org/10.1186/1939-4551-6-18

51. D'Amelio CM, Del Pozo JL, Vega O, Madamba R, Gastaminza G. Successful desensitization in a child with delayed cotrimoxazole hypersensitivity: A case report. Pediatr Allergy Immunol. 2016;27(3):320-1. http://dx.doi.org/10.1111/pai.12525

52. Chastain DB, Hutzley VJ, Parekh J, Alegro JVG. Antimicrobial desensitization: A review of published protocols. Pharmacy (Basel). 2019;7(3):112. http://dx.doi.org/10.3390/pharmacy 7030112

53. Kidon M, Blanca-Lopez N, Gomes E, Terreehorst I, Tanno L, Ponvert $C$, et al. EAACI/ENDA position paper: Diagnosis and management of hypersensitivity reactions to non-steroidal anti-inflammatory drugs (NSAIDs) in children and adolescents. Pediatr Allergy Immunol. 2018;29(5):469-80. http://dx.doi. org/10.1111/pai.12915

54. Ensina LF, de Lacerda AE, de Andrade DM, Machado L, Camelo-Nunes I, Solé D. Drug-induced anaphylaxis in children: Non-steroidal anti-inflammatory drugs and drug provocation test. J Allergy Clin Immunol Pract. 2014;2(6):825. http://dx. doi.org/10.1016/j.jaip.2014.08.016

55. Caubet JC, Rudzeviciene O, Gomes E, Terreehorst I, Brockow K, Eigenmann PA. Managing a child with possible allergy to vaccine. Pediatr Allergy Immunol. 2014;25(4):394403. http://dx.doi.org/10.1111/pai.12132

56. Kraft M, Renaudin JM, Ensina LF, Kleinheinz A, Bilò MB, Scherer Hofmeier K, et al. Anaphylaxis to vaccination and polyethylene glycol: A perspective from the European Anaphylaxis Registry. J Eur Acad Dermatol Venereol. 2021;35(10):e659-62. http://dx.doi.org/10.1111/jdv.17327.

57. Sampath V, Rabinowitz G, Shah M, Jain S, Diamant Z, Jesenak $M$, et al. Vaccines and allergic reactions: The past, the current COVID-19 pandemic, and future perspectives. Allergy. 2021;76(6):1640-60. http://dx.doi.org/10.1111/all.14840

58. Barbaud A, Deschildre A, Waton J, Raison-Peyron N, Tréchot P. Hypersensitivity and vaccines: an update. Eur J Dermatol. 2013 Apr 1;23(2):135-41. http://dx.doi.org/10.1684/ ejd.2012.1842 